OPEN ACCESS

Edited by:

Radka Stoyanova,

University of Miami, United States

Reviewed by:

Vivek Verma

University of Nebraska Medical

Center, United States

Ellen Ackerstaff,

Memorial Sloan Kettering Cancer

Center, United States

*Correspondence:

John C. Roeske

jroeske@/umc.edu

Specialty section:

This article was submitted to

Radiation Oncology,

a section of the journal

Frontiers in Oncology

Received: 01 December 2017

Accepted: 12 July 2018

Published: 31 July 2018

Citation:

Nguyen K, Haytmyradov M,

Mostafavi H, Patel R, Surucu M,

Block A, Harkenrider MM and

Roeske JC (2018) Evaluation of

Radiomics to Predict the Accuracy of

Markerless Motion Tracking of Lung

Tumors: A Preliminary Study.

Front. Oncol. 8:292.

doi: 10.3389/fonc.2018.00292

\section{Evaluation of Radiomics to Predict the Accuracy of Markerless Motion Tracking of Lung Tumors: A Preliminary Study}

\author{
Kevin Nguyen ${ }^{1}$, Maksat Haytmyradov ${ }^{2}$, Hassan Mostafavi ${ }^{3}$, Rakesh Patel ${ }^{2}$, Murat Surucu ${ }^{2}$, \\ Alec Block ${ }^{2}$, Matthew M. Harkenrider ${ }^{2}$ and John C. Roeske ${ }^{2 *}$ \\ ${ }^{1}$ Stritch School of Medicine, Loyola University Chicago, Maywood, IL, United States, ${ }^{2}$ Department of Radiation Oncology, \\ Loyola University Medical Center, Maywood, IL, United States, ${ }^{3}$ Varian, Palo Alto, CA, United States
}

Template-based matching algorithms are currently being considered for markerless motion tracking of lung tumors. These algorithms use tumor templates derived from the planning CT scan, and track the motion of the tumor on single energy fluoroscopic images obtained at the time of treatment. In cases where bone may obstruct the view of the tumor, dual energy fluoroscopy may be used to enhance soft tissue contrast. The goal of this study is to predict which tumors will have a high degree of accuracy for markerless motion tracking based on radiomic features obtained from the planning CT scan, using peak-to-sidelobe ratio (PSR) as a surrogate of tracking accuracy. In this study, CT imaging data of 8 lung cancer patients were obtained and analyzed through the open source IBEX program to generate 2,287 radiomic features. Agglomerative hierarchical clustering was used to narrow down these features into 145 clusters comprised of the highest correlation to PSR. The features among the clusters with the least inter-correlation were then chosen to limit redundancy in the data. The results of this study demonstrated a number of radiomic features that are positively correlated to PSR. The features with the highest degree of correlation included complexity, orientation and range. This approach may be used to determine patients for whom markerless motion tracking would be beneficial.

Keywords: lung cancer, motion tracking, dual energy imaging, radiomics, template matching

\section{INTRODUCTION}

It is estimated that nearly 190,000 new cases of non-small cell lung cancer (NSCLC) are diagnosed in the United States each year, accounting for $\sim 130,000$ deaths annually (1). Stereotactic body radiation therapy (SBRT) is a highly effective treatment method for early stage NSCLC patients who are medically unfit for lobectomy (2). SBRT allows for the administration of high doses of radiation to the tumor and has several advantages over conventional treatment. Moreover, it requires far fewer treatment sessions with improved local control of $\geq 90 \%$ in many studies compared to the $30-60 \%$ control rates of conventional external beam radiation therapy (EBRT) $(1,3)$.

Due to the highly conformal nature of SBRT and high dose per fraction with a limited number of fractions, motion management is critical to ensure that the tumor receives the full dose of radiation and the volume of irradiated normal tissue is minimized. To achieve these endpoints, there has 
been recent interest in lung tumor tracking during treatment delivery (4-11). The goal of these approaches is to modify the treatment (i.e., multi-leaf collimators, treatment table position, etc.), based on the position of the tumor during respiration to minimize the volume of normal tissue irradiated. This concept is critical in SBRT, as several studies have demonstrated an increased incidence of symptomatic clinical radiation pneumonitis correlating with increased volumes of normal lung irradiated (12-16). While the dosimetric and clinical results appear promising, relying on implanted markers may limit the number of patients who can receive such advanced therapies due to the risks associated with marker implantation. Previous studies with implanted fiducial markers in lung tumors demonstrate this invasive procedure carries the risk of pneumothorax and pulmonary hemorrhage (17). Moreover, migration of fiducial markers in the lung specifically may be significant (4-6).

As an alternative to using implanted markers, several studies have described using fluoroscopy for markerless tumor tracking. These studies have reported target localization $<3 \mathrm{~mm}$ using fluoroscopy and template matching methods (8-11). However, this method often fails, or results in decreased tracking accuracy, when the boundary of the tumor is obscured by overlying bones. Lewis et al. showed that the tracking error could be up to $5 \mathrm{~mm}$ due to the obstruction of the tumor by a high contrast object, such as bone (11). To address this problem, dual energy (DE) imaging has been shown to improve the visualization of tumors and in turn, improve the accuracy of markerless tumor tracking (18). Briefly, DE imaging involves obtaining $\mathrm{x}$-ray images at high (i.e., $120 \mathrm{kVp}$ ) and low (i.e., $60 \mathrm{kVp}$ ) energies. By performing a weighted-logarithmic subtraction, a third image is created that suppresses bone and enhances soft tissue/tumor.

In a recent DE study by Block et al., 74 image pairs from 17 patients were evaluated using a template matching algorithm (19). The algorithm successfully matched the template in $61(82 \%)$ of the single energy (SE) images and $74(100 \%)$ of the DE images $(p<0.01)$. The mean distance between the gross tumor volume (GTV) centroid coordinates $(x, y)$ of the matched template and physician defined ground truth coordinates was $3.2 \pm 2.8 \mathrm{~mm}$ for SE vs. $2.3 \mathrm{~mm} \pm 1.7 \mathrm{~mm}$ for $\mathrm{DE}(p=0.03)$. The false detection rate (fraction of images with $>5 \mathrm{~mm}$ matching errors) was $7 / 74(9.4 \%)$ for DE images, vs. $9 / 74(12.1 \%)$ for SE images $(p=0.79)$. It was also determined that the peak-to-sidelobe ratio (PSR)a measure of tracking quality-was predictive of template matching accuracy. For PSR $<3$, the matching accuracy was $3.3 \pm 3.1 \mathrm{~mm}$ vs. $2.2 \pm 1.3 \mathrm{~mm}$ for PSR $\geq 3(p=0.02)$. Importantly, the false detection rate was $20.9 \%($ PSR $<3$ ) vs. $4.0 \%$ (PSR $\geq 3)(p<0.01)$. Therefore, the PSR can be used as a predictive surrogate for the accuracy of template matching.

The goal of this study is to determine if one can predict which tumors will have a high degree of accuracy for markerless motion tracking using radiomic features obtained from the planning CT scan. Radiomics is the high throughput extraction and analysis of quantitative features from imaging data (20). These quantitative features have prognostic and predictive potential containing information that cannot be obtained via inspection of the imaging data alone $(21,22)$. The information found from these features is multidimensional and can comprise of anything from tumor malignancy to predicting patient outcomes $(23,24)$. To our knowledge, this study represents the first to consider the use of radiomic parameters to predict the success of a markerless motion tracking technique.

\section{MATERIALS AND METHODS}

\section{Patient Selection}

In this Loyola University Medical Center Institutional Review Board (IRB) approved study (LU203840), SBRT patients with Stage IA-IIA NSCLC were enrolled in a prospective imaging study. Patients who were eligible for this study included those with no implanted fiducials and a Karnofsky Performance Status (KPS) $>70$. In total, 8 patients were enrolled and a total of 23 imaging fractions were evaluated.

\section{CT Simulation and Treatment Planning}

Patients were simulated in the supine position, and immobilized using a custom body mold (Alpha Cradle, Smithers Medical Products, Inc., Canton, $\mathrm{OH}$ ) that was indexed to the treatment table. Simulation was performed using a dedicated CT scanner (Brilliance Large Bore, Philips Medical Systems, Andover, MA) equipped with the Real-Time Position Management (RPM) System (Varian Medical Systems, Palo Alto, CA) to allow for $4 \mathrm{D}$ acquisitions. A slice thickness of $3 \mathrm{~mm}$ was used for all patients. The tumor volume and prescription were determined by the treating radiation oncologist. Treatment plans, using a volumetric modulated arc therapy (VMAT) technique were created in conjunction with the dosimetry team and ultimately approved by the radiation oncologist.

\section{Fluoroscopic Image Acquisition}

All patients were treated on a Varian TrueBeam (Varian Medical Systems, Inc., Palo Alto, CA) linear accelerator equipped with an on-board imaging system and RPM. Following each fraction, fluoroscopic images were obtained sequentially using 60 and $120 \mathrm{kVp}$, respectively. These energies were based on previous studies in which the radiographic technique was optimized for $\mathrm{DE}$ subtraction $(25,26)$. The image sequences were acquired at fixed gantry angles along the same trajectory that was used for VMAT delivery. The Varian TrueBeam Integrated Imaging Solution ${ }^{\circledR}$ in conjunction with the Varian iTools Capture (Varian Medical Systems) software and a Matrox Imaging (Quebec, Canada) frame grabber card were used to acquire a total of 2,448 fluoroscopy frames.

\section{Dual Energy Subtraction}

The raw images, obtained from the iTools Capture software, were exported off-line into a customized program developed in MATLAB (MathWorks, Natick, MA). Alignment of the individual fluoroscopic frames (60 and $120 \mathrm{kVp}$ ) was performed based on the image amplitude and phase as obtained by RPM. Further refinements were made using a localized rigid 
registration (27). Based on the aligned images, a pixel-by-pixel, weighted logarithmic subtraction was performed to create the DE image using $(25,26)$ :

$$
\ln \left(I^{D E_{\text {Soft }}}\right)=\ln \left(I^{\text {High }}\right)-w_{s} \ln \left(I^{\text {Low }}\right)
$$

where $\mathrm{I}_{\mathrm{Soft}}^{\mathrm{DE}}$ is the intensity of the resultant DE soft tissue (bone suppressed) image, $\mathrm{I}^{\text {High }}$ and $\mathrm{I}^{\text {Low }}$ are the intensities the high and low energy image pixels, respectively, $\ln$ is the natural logarthim and $\mathrm{w}_{\mathrm{s}}$ is the relative weight required to produce the $\mathrm{DE}$ image. The $120 \mathrm{kVp}$ images were used as the standard SE images to which the DE images were compared.

\section{Template Matching}

Template matching was performed using an algorithm based on Mostafavi et al. (28). First, templates are generated from the CT where the radiation oncologist contours the GTV during routine RT planning. The program then creates a volume of interest (VOI) around the GTV and the voxel values within this volume are forward projected to create templates that are similar to digitally reconstructed radiographs (DRRs). Templates are generated for all expected treatment angles and the appropriate template is selected based on imaging angle (28). Next, the match location is determined by shifting the template across the image and calculating the normalized cross-correlation (NCC) between the template and the acquired image at different $2 \mathrm{D}$ offsets within the search region. The NCC is given by (28):

$$
N C C=\frac{1}{n} \sum_{x, y} \frac{(f(x, y)-f)(t(x, y)-t)}{\sigma_{f} \sigma_{t}}
$$

where $n$ is the number of pixels in the template $t_{(\mathrm{x}, \mathrm{y})}$ and subimage $f_{(\mathrm{x}, \mathrm{y})}, t$ and $f$ are the average of the pixel values in the template and sub-image, respectively, while $\sigma_{f}$ and $\sigma_{t}$ are the standard deviations over the respective regions. Calculation of NCC at different $2 \mathrm{D}$ offsets within a search region results in a match score surface. The offset at which NCC has the maximum value (i.e., the position of the peak of the match score surface) represents the potential target position within the search region. The strength of this peak relative to NCC values away from the peak, called sidelobe values, is quantified by the peak-to-sidelobe ratio (PSR) and is calculated by (28):

$$
P S R=\frac{[\text { peakvalue }- \text { meansidelobe }]}{[\text { sidelobe standard deviation }]}
$$

\section{Radiomic Analysis}

For the study, CT imaging data from 8 lung cancer patients treated with SBRT was obtained from Eclipse treatment planning system (Varian Medical Systems, Palo Alto, CA) and analyzed using the open source IBEX program (28). The GTV, as outlined on the $50 \%$ respiratory phase CT was the ROI chosen for all of the patients and imaging features of the following feature sets were calculated: Gray Level Run Matrix 25 ( $n=33$ features),
Intensity Direct ( $n=55$ features), Intensity Histogram ( $n=$ 49 features), Neighbor Intensity Difference 3 ( $n=5$ features), Neighbor Intensity Difference 25 ( $n=5$ features), Gray Level Co-occurrence Matrix 3 ( $n=1792$ features), Gray Level Cooccurrence Matrix 25 ( $n=330$ features), and Shape $(n=18$ features). A summary of these feature sets is presented in Table II of Zhang et al. (29). Of note, the number of features listed here may not be consistent with Zhang et al. (29). The reason is that certain features (such as Percentile) will take on discrete values that are not specifically listed in the table.

A total of 2,287 imaging features were identified in initial dataset for all 8 patients. Imaging features that were undefined or missing for certain patients were removed from the dataset. Additionally, some imaging features from Intensity Direct and Intensity Histogram sets contained duplicate entries, which were subsequently removed. After the pre-processing, the number imaging features decreased to 1,419 .

The study aimed to find a subset of imaging features most predictive in predicting PSR values. In this analysis, PSR values were used as a continuous variable. To find a subset of features that is relevant in predicting PSR, initially, agglomerative hierarchical clustering was performed based on the Pearson correlation coefficient between features. Hierarchical clustering was selected for being a simple, straightforward clustering measure with easily interpretable results. Agglomerative hierarchical clustering finds clusters by iteratively merging the two most similar features, or groups of features, together. The features with highest correlation coefficients are grouped together first. The results of hierarchical clustering can be presented as a dendogram seen in Figure 1, where the number of clusters depends on what level the dendogram tree is cut. This particular cluster has correctly identified features that fall into the Gray Level Co-occurrence Matrix 3 feature set.

The optimal number of clusters was chosen by iteratively decreasing the cluster size and evaluating the Dunn index (30). The Dunn index is defined as the ratio between closest intercluster distances and the furthest intra-cluster distance. The largest value of the Dunn index provides the optimal number of partitions in the data. Using this procedure, we found the optimal number of feature clusters to be 145 . This is demonstrated in Figure 2.

Among each cluster set we chose the feature that is least correlated to the rest of clusters. This allows for the reduction of noise and removal of redundancy. In the next step, we use target values of PSR and perform linear regression with squared regularization term. For the penalty term, we chose an intermediate value of 0.6 to avoid over-fitting. Feature ranking was performed based on the normalized absolute values of the weights obtained by linear regression.

\section{Validation Studies}

Additionally, validation studies for the results were conducted to ensure that this algorithm was shown to be able discover features that are predictive of tracking. Four categories of feature groups were assessed: (1) features selected by agglomerative hierarchical clustering, (2) all 2,287 features together (3) top four 


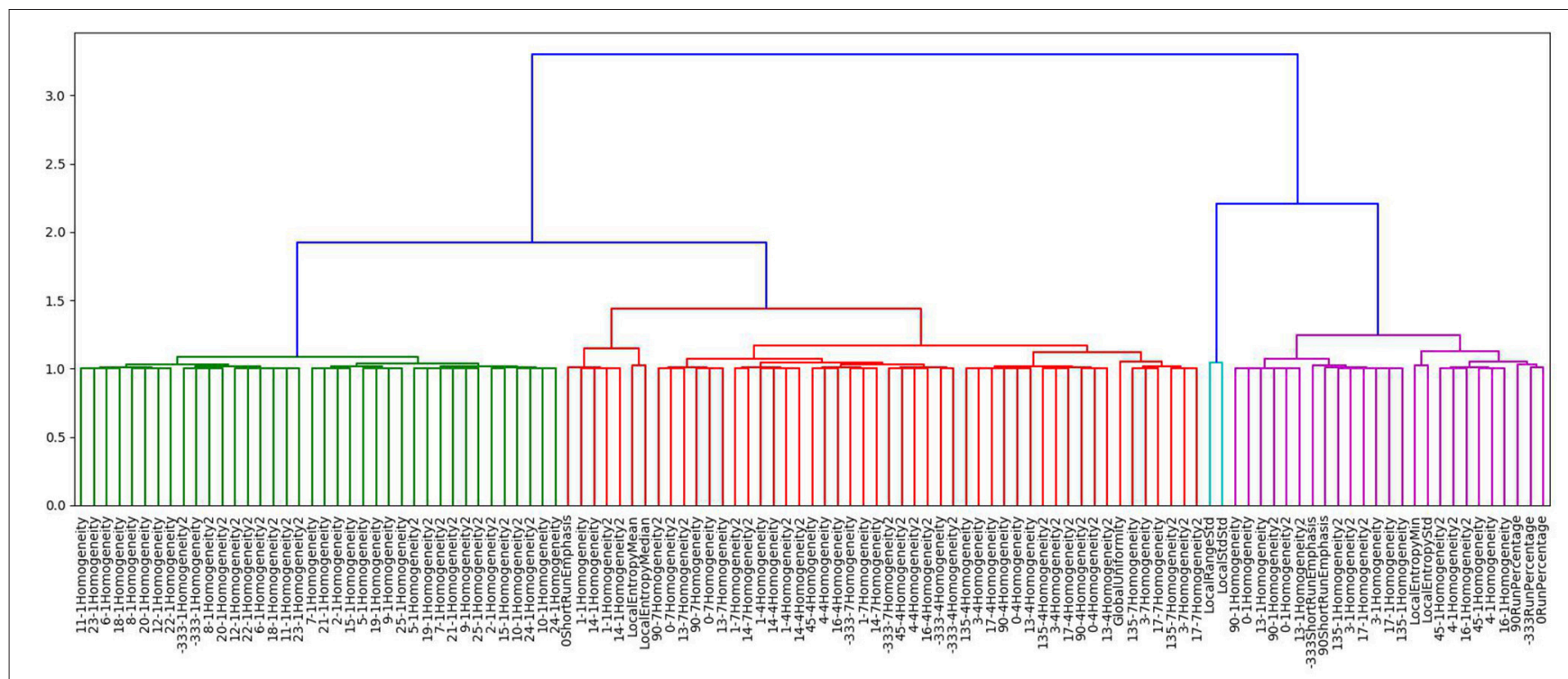

FIGURE 1 | Dendogram generated using agglomerative hierarchal clustering showing just one of the clusters formed based on this method. Depending on the level of the clustering chosen, the number of clusters formed is variable.

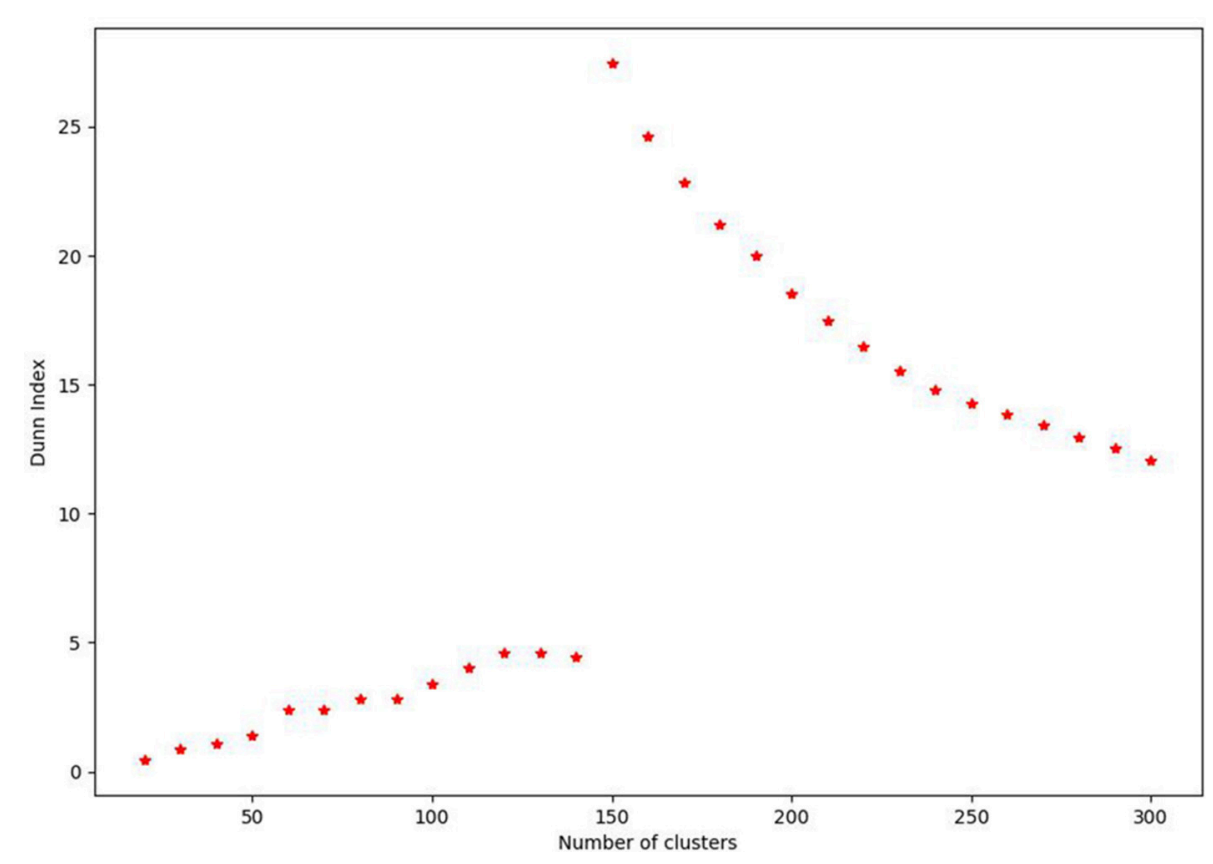

FIGURE 2 | Plot demonstrating calculation of the Dunn Index for the agglomerative hierarchal clustering performed for the radiomic features of this study. From 0 to 144 , there is a gradual increase in the Dunn Index before a marked increase at 145 followed by decreasing values. This indicates that 145 clusters are the optimal value for this data set.

features from the subset of agglomerative clustering, and (4) top nine features solely selected based on Pearson correlation. For each category the data was randomly split into $50 \%$ training and $50 \%$ testing samples. Ridge regression was done to perform linear fit on the training sample and predict the PSR of the testing sample. For each split, the r-square score was computed for the testing sample and it was repeated five times. The mean $r$-square of five splits was compared for three categories. The r-square can range between 1 to arbitrary negative values; 1 signifies the features are able to generalize to the dataset and negative values imply the model can be arbitrarily worse. 


\section{RESULTS}

The study evaluated 1,419 radiomic features for 8 patients which was narrowed down using agglomerative hierarchal clustering to 145 clusters. From there, the 20 features with the strongest relationship to the PSR value are summarized in Figure 3, 4. In the analysis of the radiomics features, it was found that there were several features that showed high predictive potential for PSR. The features complexity, orientation, gray level non-uniformity and range demonstrated the highest degree of correlation with PSR for both SE and DE imaging. Other radiomic features with high correlation coefficients are also included as well. Of note, many of the intensity based features demonstrated high correlation, including: energy,

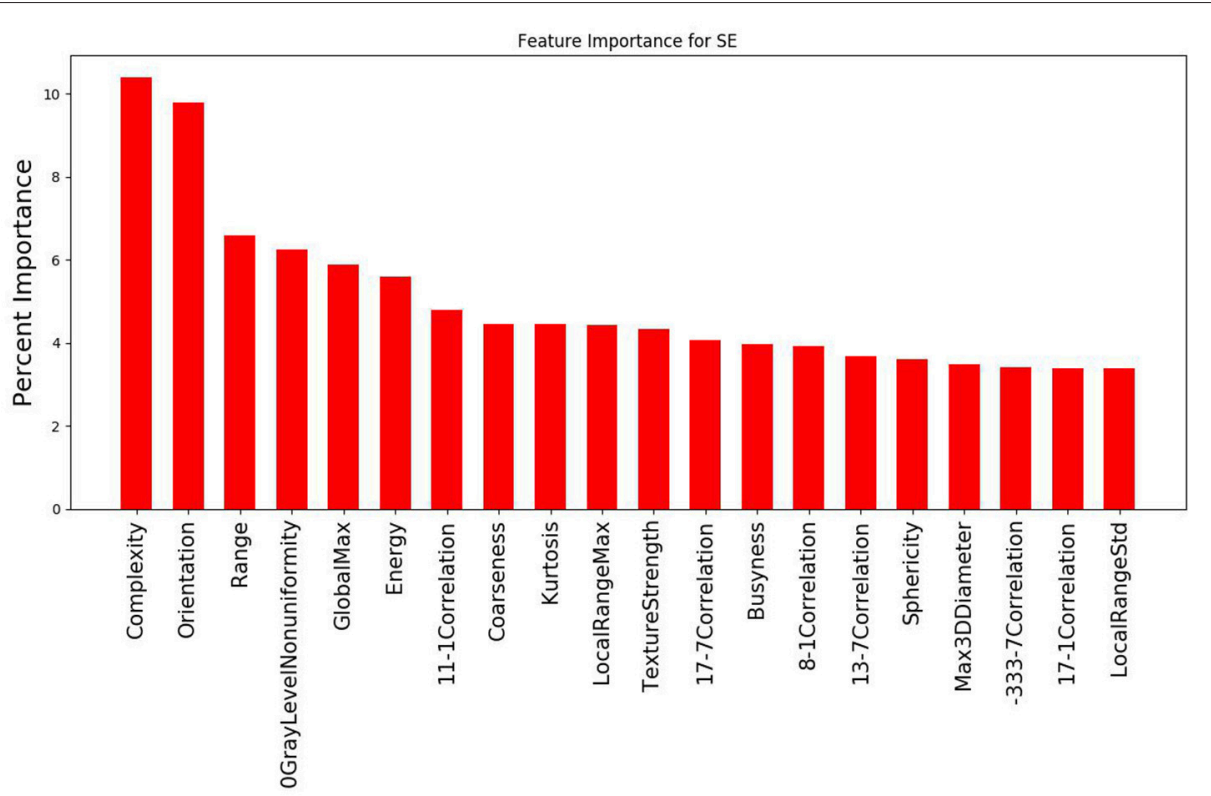

FIGURE 3 | Graph demonstrating the 20 features with the strongest relationship to PSR for the SE imaging data out of the 145 cluster sets. Complexity and orientation were the most predictive of PSR for SE.

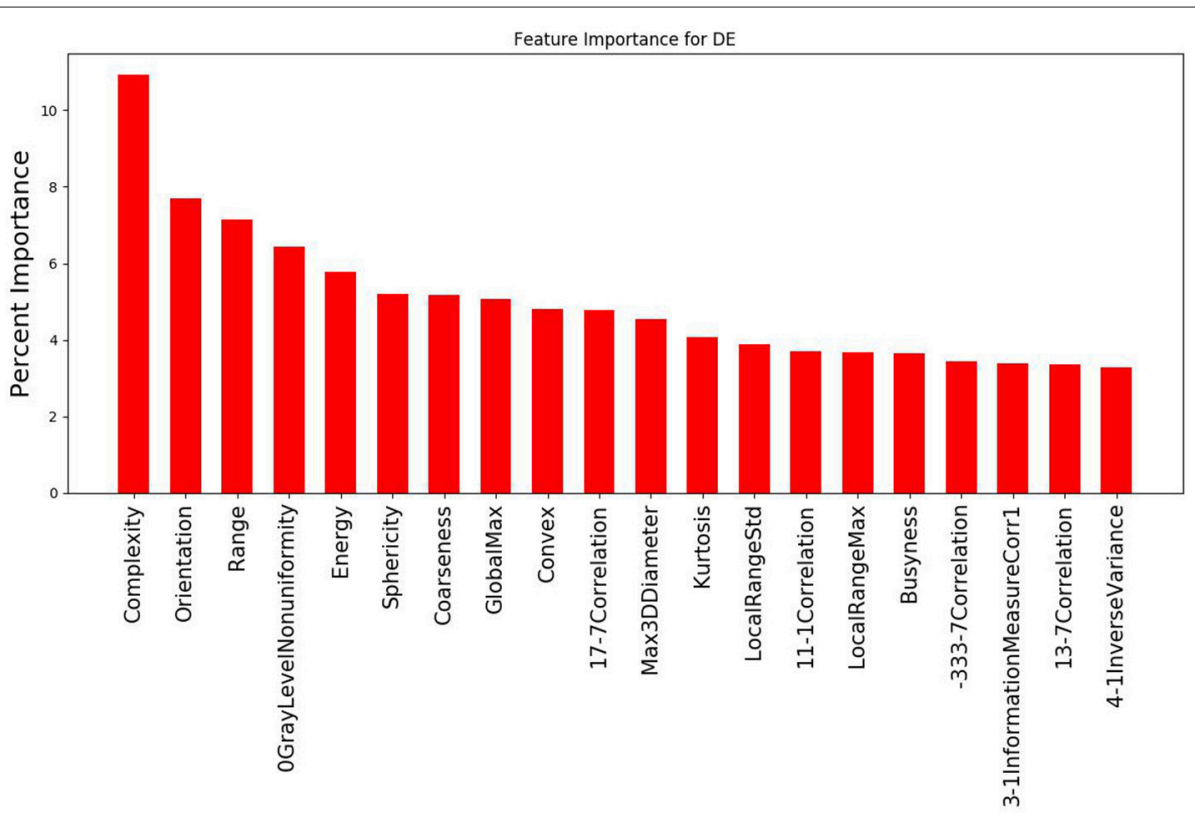

FIGURE 4 | Graph demonstrating the 20 features with the strongest relationship to PSR for the DE imaging data out of the 145 cluster sets. Complexity and orientation were the most predictive of PSR for DE, similar to the results for SE. 
globalmax, kurtosis, localrangemax, and localrangestd. SE and DE demonstrated similar results in regards to correlative features. Of all the radiomic features highlighted, all $p$-values were $<0.05$ indicating statistical significance of the correlation of the feature to the SE and DE PSR values.

The validation study revealed that the mean r-square of features in Category 1 (those selected by the method used in the study) were higher than in Category 2, 3, or 4. That is, the features selected by the method in the study are able to generalize the data to a better degree than all 2,287 features together or single features alone. Category 1 had a r-square of $0.29+/-0.18$ while Category 2 had a r-square of -3.24 $+/-2.32$. Category 3 , consisting of only the top four features from the selected feature set, had a r-square score $-4.13+/-$ 8.62. Category 4 consisted of single features and a sampling of the features with highest correlation shows that SurfaceArea, 333GrayLevelNonuniformity and Energy had a r-square of 0.08 $+/-0.36,-0.1+/-0.63$, and $-0.14+/-0.51$, respectively. Based on this validation for the features selected in this study, Category 1 demonstrates better prediction that either the remaining categories.

\section{DISCUSSION}

In this study, we evaluated the correlation between radiomic parameters and the accuracy of a template-based markerless motion tracking algorithm. Overall, a high correlation coefficient was observed between several radiomic features and the PSR values for both SE and DE images. The significance of these results is that PSR $>3$ have been shown to have improved tracking accuracy. Thus, any features correlated with the PSR value would be able to indicate the potential for quality tracking. In turn, this may allow for the identification of patients which are ideal candidates for using the proposed markerless motion tracking algorithm during SBRT delivery.

For both SE and DE imaging, the top 3 features with the strongest relationship to the PSR were identical: complexity, orientation, and range. Complexity is a feature of the neighbor intensity difference category. Complexity is determined by the amount of primitive components within an image, making it non-uniform due to rapid change in the gray level intensity. Increased complexity can potentially increase the ability to track heterogeneous tumors, which will likely have more complexity compared to normal healthy tissues, which should be more regular. Orientation is a feature of the shape category related to the directionality of the region of interest (ROI), which is reflective of the shape of a tumor. Range is a feature of the intensity direct category that represents the range of gray values in the ROI. This is expected to have a large effect on the ability

\section{REFERENCES}

1. Siegel R, Miller K, Jemal A. Cancer Statistics, 2017. CA. Cancer J. Clin (2017) 67:7-30. doi: 10.3322/caac. 21387

2. Timmerman R, Paulus R, Galvin J. Stereotactic body radiation therapy for inoperable early stage lung cancer. JAMA (2010) 303:1070-6. doi: $10.1001 /$ jama.2010.261 to track in a similar fashion to complexity. A wider range of gray values will more likely indicate heterogeneity, which serves as a good contrast against more homogenous healthy tissue.

There were several limitations in the scope of this study with the key factor being the small sample size of only 8 patients. Sample size is an important variable is assessing the predictive value of a particular set of radiomic parameters. A small sample size has a larger margin of selection bias, which would mean that the conclusions from this study are not as widely applicable as desired. Zhang et al. (31) demonstrated that a patient cohort of $>50$ is needed for convergence of the area-under-the curve (AUC) in their analysis. Figueroa et al. (32) predicted a sample size ranging from 80 to 560 is required to reduce the root-meansquare error to $<0.01$. Moreover, it is not clear how the size of the patient cohort would affect the clustering of radiomic parameters. The next step forward for this study would be applying what was learned here to a larger patient cohort in the aims of corroborating these findings. There are some additional challenges to the process of radiomics as results may differ institutionally or even on different imaging devices. One of the main goals currently in radiomics is standardization of imaging acquisition to ensure consistent and reliable results (33).

This study demonstrated several radiomic features that are positively correlated to PSR, an indicator of how well markerless motion tracking will function for a specific tumor in a patient. Based on the predictive relationship between the PSR value and the radiomic features, ultimately the goal is to use CT imaging data to determine if a patient would make a good candidate for markerless motion tracking SBRT. Radiomics thus offers tremendous potential in improving individualized treatment plans.

\section{AUTHOR CONTRIBUTIONS}

RP, MS, AB, MMH, and JR contributed to the acquisition of this clinical data used in this study. $\mathrm{KN}, \mathrm{MH}$, and $\mathrm{HM}$ contributed to the data analysis and methodology. $\mathrm{KN}$ and $\mathrm{MH}$ performed the radiomic analysis and machine learning used in this analysis, as well as drafted the manuscript. All co-authors contributed to the review and editing of the manuscript. JR was the senior author and supervised all aspects of the project.

\section{FUNDING}

Research reported in this publication was supported by the National Cancer Institute of the National Institutes of Health under Award Number R01CA207483. The content is solely the responsibility of the authors and does not necessarily represent the official views of the National Institutes of Health.
3. Fang LC, Komaki R, Allen P, Guerrero T, Mohan R, Cox J. Comparison of outcomes for patients with medically inoperable stage I non-small-cell lung cancer treated with two-dimensional vs. three-dimensional radiotherapy. Int J Radiat Oncol Biol Phys. (2006) 66:108-16. doi: 10.1016/j.ijrobp.2006. 04.015

4. Nuyttens JJ, Prévost JB, Praag J, Hoogeman M, Van Klaveren RJ, Levendag PC, et al. Lung tumor tracking during stereotactic radiotherapy treatment with the 
CyberKnife: marker placement and early results. Acta Oncol. (2006) 45:961-5. doi: 10.1080/02841860600902205

5. Shirato H, Harada T, Harabayashi T, Hida K, Endo H, Kitamura K, et al. Feasibility of insertion/implantation of $2.0-\mathrm{mm}$-diameter gold internal fiducial markers for precise setup and real-time tumor tracking in radiotherapy. Int J Radiat Oncol Biol Phys. (2003) 56:240-7.

6. Nelson C, Starkschall G, Balter P, Morice RC, Stevens CW, Chang JY. Assessment of lung tumor motion and setup uncertainties using implanted fiducials. Int J Radiat Oncol Biol Phys. (2007) 67:915-23. doi: 10.1016/j.ijrobp.2006.10.033

7. Hoisak JD, Sixel KE, Tirona R, Cheung PC, Pignol JP. Correlation of lung tumor motion with external surrogate indicators of respiration. Int $J$ Radiat Oncol Biol Phys. (2004) 60:1298-306. doi: 10.1016/j.ijrobp.2004.07.681

8. Xu Q, Hamilton RJ, Schowengerdt RA, Alexander B, Jiang SB. Lung tumor tracking in fluoroscopic video based on optical flow. Med Phys. (2008) 35:5351-9.

9. Lin T, Cerviño LI, Tang X, Vasconcelos N, Jiang SB. Fluoroscopic tumor tracking for image-guided lung cancer radiotherapy. Phys Med Biol. (2009) 54:981-92. doi: 10.1088/0031-9155/54/4/011

10. Rottmann J, Aristophanous M, Chen A, Court L, Berbeco R. A multi-region algorithm for markerless beam's-eye view lung tumor tracking. Phys Med Biol. (2010) 55:5585-98. doi: 10.1088/0031-9155/55/18/021

11. Lewis JH, Li R, Watkins WT, Lawson JD, Segars WP, Cerviño LI, et al. Markerless lung tumor tracking and trajectory reconstruction using rotational cone-beam projections: a feasibility study. Phys Med Biol. (2010) 55:2505-22. doi: 10.1088/0031-9155/55/9/006

12. Graham MV, Purdy JA, Emami B, Harms W, Bosch W, Lockett MA, et al. Clinical dose-volume histogram analysis for pneumonitis after 3D treatment for non-small cell lung cancer (NSCLC). Int J Radiat Oncol Biol Phys. (1999) 45:323-9.

13. Rodrigues G, Lock M, D'Souza D, Yu E, Van Dyk J. Prediction of radiation pneumonitis by dose - volume histogram parameters in lung cancer - a systematic review. Radiother Oncol. (2004) 71:127-38. doi: 10.1016/j.radonc.2004.02.015

14. Schallenkamp JM, Miller RC, Brinkmann DH, Foote T, Garces YI. Incidence of radiation pneumonitis after thoracic irradiation: dosevolume correlates. Int J Radiat Oncol Biol Phys. (2007) 67:410-6. doi: 10.1016/j.ijrobp.2006.09.030

15. Barriger RB, Forquer JA, Brabham JG, Andolino DL, Shapiro RH, Henderson $\mathrm{MA}$, et al. A dose-volume analysis of radiation pneumonitis in non-small cell lung cancer patients treated with stereotactic body radiation therapy. Int J Radiat Oncol Biol Phys. (2012) 82:457-62. doi: 10.1016/j.ijrobp.2010. 08.056

16. Matsuo Y, Shibuya K, Nakamura M, Narabayashi M, Sakanaka K, Ueki N, et al. Dose-volume metrics associated with radiation pneumonitis after stereotactic body radiation therapy for lung cancer. Int J Radiat Oncol Biol Phys. (2012) 83:e545-9. oi:10.1016/j.ijrobp.2012.01.018.

17. Trumm CG, Haussler SM, Muacevic A, Stahl R, Stintzing S, Paprottka PM, et al. CT fluoroscopy-guided percutaneous fiducial marker placement for CyberKnife stereotactic radiosurgery: technical results and complications in 222 consecutive patients. J Vasc Interv Radiol. (2014) 25:760-8. doi: 10.1016/j.jvir.2014.01.004

18. Patel R, Panfil J, Campana M, Block AM, Harkenrider M, Surucu M, Roeske JC. Markerless motion tracking of lung tumors using dual-energy fluoroscopy. Med Phys. (2015) 42:254-62.

19. Block AM, Patel R, Surucu M, Harkenrider MM, Roeske JC. Evaluation of a template-based algorithm for markerless lung tumour localization on single- and dual-energy kilovoltage images. Br J Radiol. (2016) 89:20160648. doi: 10.1259/bjr.20160648
20. Lambin P, Rios-Velazquez E, Leijenaar R, Carvalho S, van Stiphout RG, Granton P, et al. Radiomics: extracting more information from medical images using advanced feature analysis. Eur J Cancer (2012) 48:441-6. doi: 10.1016/j.ejca.2011.11.036

21. Lambin P, van Stiphout RG, Starmans MH, Rios-Velazquez E, Nalbantov G, Aerts HJ, et al. Predicting outcomes in radiation oncologymultifactorial decision support systems. Nat Rev Clin Oncol. (2013) 10:27-40. doi: 10.1038/nrclinonc.2012.196

22. Lambin P, Roelofs E, Reymen B, Velazquez ER, Buijsen J, Zegers CM, et al 'Rapid Learning health care in oncology' - an approach towards decision support systems enabling customised radiotherapy. Radiother Oncol. (2013) 109:159-64. doi: 10.1016/j.radonc.2013.07.007

23. Aerts HJ, Velazquez E, Leijenaar R, Parmar C, Grossmann P, Carvalho S, et al. Decoding tumour phenotype by noninvasive imaging using a quantitative radiomics approach. Nat Commun. (2014) 5:4006. doi: 10.1038/ncomms5006

24. Atwater T, Balagurunathan Y, Antic S, Chen H, Schabath M, Gu Y, et al Radiomic analysis for improved lung cancer prediction of indeterminate pulmonary nodules. Am J Respir Crit Care Med. (2015) 191:A6119.

25. Hoggarth MA, Luce J, Syeda F, Bray TS, Block A, Nagda S, et al. Dual energy imaging using a clinical on-board imaging system. Phys Med Biol. (2013) 58:4331-40. doi: 10.1088/0031-9155/58/12/4331

26. Sherertz T, Hoggarth M, Luce J, Block AM, Nagda S, Harkenrider MM, et al. Prospective evaluation of dual-energy imaging in patients undergoing image guided radiation therapy for lung cancer: initial clinical results. Int J Radiat Oncol Biol Phys. (2014) 89:525-31. doi: 10.1016/j.ijrobp.2014.03.004

27. Balter HM, Pelizzari CA, Chen GT. Correlation of projection radiographs in radiation therapy using open curve segments and points. Med Phys. (1992) 19:329-34.

28. Mostafavi H, Sloutsky A, Jeung A. Detection and localization of radiotherapy targets by template matching. In: 2012 Annual International Conference of the IEEE Engineering in Medicine and Biology Society. San Diego, CA: IEEE (2012). p. 6023-27.

29. Zhang L, Fried DV, Fave XJ, Hunter LA, Yang J, Court LE, et al. IBEX: An open infrastructure software platform to facilitate collaborative work in radiomics. Med Phys. (2015) 42:1341-53. doi:10.1118/1.4908210

30. Dunn, CJ. Well-separated clusters and optimal fuzzy partitions. J Cyber (1973) 4:95-104. doi: 10.1080/01969727408546059

31. Zhang Y, Oikonomou A, Wong A, Haider MA, Khalvati F. Radiomics-based prognosis analysis for non-small cell lung cancer. Sci Rep. (2017) 7:46349. doi: $10.1038 /$ srep 46349

32. Figueroa RL, Zeng-Treitler Q, Kandula S, Ngo LH. Predicting sample size required for classification performance. BMC Med Inform Decis Mak. (2012) 12:8. doi: 10.1186/1472-6947-12-8

33. Kumar V, Gu Y, Basu S, Berglund A, Eschirich SA, Schabath MB, et al. Radiomics: the process and the challenges. Magn Reson Imaging (2012) 30:1234-48. doi: 10.1016/j.mri.2012.06.010

Conflict of Interest Statement: Loyola University has a Master Research Agreement with Varian Medical Systems. The authors declare that the research was conducted in the absence of any commercial or financial relationships that could be construed as a potential conflict of interest.

Copyright (c) 2018 Nguyen, Haytmyradov, Mostafavi, Patel, Surucu, Block, Harkenrider and Roeske. This is an open-access article distributed under the terms of the Creative Commons Attribution License (CC BY). The use, distribution or reproduction in other forums is permitted, provided the original author(s) and the copyright owner(s) are credited and that the original publication in this journal is cited, in accordance with accepted academic practice. No use, distribution or reproduction is permitted which does not comply with these terms. 